\title{
VARIATIONAL CALCULATIONS OF MODELS OF NUCLEAR AND NEUTRON MATTER $\dagger$
}

\author{
I. E. LAGARIS, V. R. PANDHARIPANDE and R. B. WIRINGA \\ Department of Physics, University of Ilinois at Urbana-Champaign, Urbana, IL 61801
}

Received 23 February 1979

\begin{abstract}
Abetract: We report results of variational calculations of models of nuclear matter in which the nuclear interaction is approximated by a sum of central, spin, isospin and tensor forces. The models are based on realistic potentials such as those of Reid, Bethe-Johnson, Hamada-Johnston, and Gammel-Thaler. The correlation operator in the variational wave function contains central, spin, isospin and tensor terms. We briefly review the Fermi-hypernetted-chain, and single-operatorchain (SOC) methods used to calculate the energy expectation value. The energies obtained for these simple models by various variational and reaction matrix calculations seem to be in reasonable agreement. Results with the SOC approximation for the $v_{3}$ model of neutron matter, in which the interaction has only central and spin components, are also reported. These are in good agreement with the energies obtained by summing multiple operator chains.
\end{abstract}

\section{Introduction}

Variational theories of nuclear matter are generally based on the Hamiltonian:

$$
H=\sum_{i}-\frac{\hbar^{2}}{2 m} \nabla_{l}^{2}+\sum_{i<j} \sum_{p} v^{p}\left(r_{i j}\right) O_{i}^{p}
$$

where $v^{p}\left(r_{i j}\right)$ are functions of $\left|r_{i}-r_{j}\right|$, and $O_{i j}^{p}$ are operators. In principle we must take as many operators $O_{i j}^{p}$ as are required to explain the NN scattering data at non-relativistic energies. However we will consider only the following eight operators:

$$
O_{i j}^{p-1,8}=1, \sigma_{i} \cdot \sigma_{j}, \tau_{i} \cdot \tau_{b}\left(\sigma_{i} \cdot \sigma_{j}\right)\left(\tau_{i} \cdot \tau_{j}\right), S_{i b}, S_{l j}\left(\tau_{i} \cdot \tau_{j}\right),(\boldsymbol{L} \cdot S)_{i,} \text { and }(\boldsymbol{L} \cdot S)_{i j}\left(\tau_{i} \cdot \tau_{i}\right) \text {, }
$$

where $S_{i j}$ and $(\boldsymbol{L} \cdot S)_{i j}$ are the tensor and spin-orbit operators. For convenience we will occasionally use the superscripts $c, \sigma, \tau, \sigma \tau, t, t \tau, b$ and $b \tau$ instead of $p=1,8$ to denote the $O^{p}, v^{p}$ etc.

At least two more operators, containing quadratic spin-orbit terms, and possibly many more are required to describe realistic potentials in this fashion. Models of nuclear matter based on Hamiltonians that neglect some of the operator dependences of the potential are popularly called homework models. The simplest of these,

† Supported by NSF PHYS 76-22147. 
such as $v_{1}$ and $v_{2}$ have only a central potential ${ }^{1}$ ), while $v_{3}$ has a central plus a $\sigma_{1} \cdot \sigma_{2}$ potential and is used for neutron matter ${ }^{2}$ ). The models $v_{6}$ and $v_{8}$ respectively have the operators $O_{i j}^{p-1,6}$ and $O_{i j}^{p-1,8}$ in their Hamiltonians.

We hope to calculate the equation of state $E(\rho)$ of nuclear matter with a correlation operator:

$$
F_{i j}=\sum_{p=1, n} f^{p}\left(r_{i j}\right) O_{i,}^{p}
$$

and a variational wave function

$$
\Psi_{v}=\left(\mathscr{S} \prod_{i<i} \mathscr{F}_{i j}\right) \Phi
$$

Here $\Phi$ is the Fermi-gas wave function, and the product of $\mathscr{F}_{i j}$ is symmetrized because the $\mathscr{F}_{y}$ do not commute. The $f^{\sigma}, f^{\tau}$ and $f^{\sigma \tau}$ partly simulate the $l$-dependence of the correlation due to backflow ${ }^{3}$ ) and are non-zero even when $v^{\sigma}, v^{\tau}$ or $v^{\sigma \tau}$ are zero as in the $v_{1}$ and $v_{2}$ models. The tensor and spin-orbit correlations are generated respectively by tensor and spin-orbit forces. Thus in eq. (1.3) $n=4$ in models $v_{1}, v_{2}$ and $v_{3}$, while $n=6,8$ in models $v_{6}$ and $v_{8}$ respectively.

The $v_{2}$ model has been studied with a more general wave function that contains three-body and state-dependent two-body correlations. ${ }^{3}$ ) In this case the threebody correlation is found to be negligible, but the state-dependent correlation is not. The $f^{\sigma}, f^{\top}$ and $f^{\sigma \tau}$ can simulate the correct state-dependence of correlations only at small momenta. In the $v_{2}$ model the $E(\rho)$ calculated with (1.4) is found to be $\simeq 0.5-3.0 \mathrm{MeV}$ too high in the density range $k_{\mathrm{F}}=1.3-2.0 \mathrm{fm}^{-1}$. This provides some indication of the effect of neglecting momentum-dependent terms in the correlation operator (1.3). How well the wave function (1.4) does in describing the many-body spin, isospin, tensor etc. correlations is a very open question.

In principle the $f^{p}$ should be obtained by minimizing the energy by generalizing the methods being developed by Lantto and Siemens. ${ }^{4}$ ) However, near equilibrium the $E(\rho)$ is not very sensitive to the specific choice of $f^{p}$, and so we follow an approximate procedure. A set of $\mathscr{F}_{i /}$ described by parameters $d$ and $\beta_{p>1}$ :

$$
\mathscr{F}_{i j}\left(d, \beta_{p>1}\right)=f^{c}\left(d, r_{i j}\right)+\sum_{p=2, n} \beta_{p} f^{p}\left(d, r_{i j}\right) O_{i p}^{p}
$$

is used to calculate the $E\left(\rho, d, \beta_{p>1}\right)$. The functions $f^{p}(d, r)$ are obtained by minimizing the contribution of two-body clusters to the energy, under "healing" constraints

$$
\mathscr{F}(r>d)=1, \quad \nabla F(r=d)=0,
$$

meant to simulate variation of the many-body cluster contributions $\left.{ }^{5.6}\right)$. The optimum healing distance is determined variationally. Eq. (1.6) implies $f^{p>1}(r>d)=0$, and the parameters $\beta_{p>1}$ simply vary the magnitudes of the $f^{p>1}(r)$ in $F$; since 
$f^{f}(r>d)=1$, there is no $\beta_{\mathrm{c}}$. The $f^{p}(d, r<d)$ satisfy coupled Schrödinger-type equations ${ }^{7}$ ) that are easy to solve.

\section{The chain summations}

The numerator and the denominator of the energy expectation value:

$$
E=\frac{\left\langle\Phi^{*}\left(\mathscr{S} \prod_{i<j} \mathscr{F}_{l j}^{+}\right)|H|\left(\mathscr{S} \prod_{i<j} \mathscr{F}_{l i}\right) \Phi\right\rangle}{\left\langle\Phi^{*}\left(\mathscr{S} \prod_{i<j} \mathscr{F}_{i j}^{+}\right) \mid\left(\mathscr{S} \prod_{i<j} \mathscr{F}_{i j}\right) \Phi\right\rangle}
$$

are expanded in powers of short-range functions $F^{D}\left(r_{i l}\right)$,

$$
F^{\infty}(r)=f^{c^{2}}(r)-1, \quad F^{p>1}(r)=2 f^{c}(r) f^{p}(r),
$$

and $f^{p>1}(r) f^{q>1}(r)$. The resulting terms are represented diagrammatically using the dictionary of elements shown in fig. 1 . We will refer to elements 1-3 of fig. 1 as correlation lines, and elements $6-8$ as derivative lines. Element 5 is called an interaction line, and

$$
f^{p} H^{a} f^{\prime}=f^{p}(r)\left(-\frac{\hbar^{2}}{m} \nabla^{2} f^{\prime}(r) \delta_{q c}+v^{q}(r) f^{\prime}(r)\right) .
$$

Detailed diagram rules can be found in refs. ${ }^{7.8}$ ); the following discussion is meant merely to clarify the notation.

Briefly the diagrams contain two or more points representing coordinates $r_{i}$. Each point of a denominator diagram must be connected to another by a correlation line. Numerator diagrams are divided into four classes $W, U, W_{\mathrm{F}}$ and $U_{\mathrm{F}}$. The $W$ diagrams must contain one interaction line $m n$, while the $U$-diagrams have two derivative lines $m n$ and $m o$. The $W_{F}\left(U_{F}\right)$ diagrams have a derivative line $m n$ and a derivative state line (element 9 of fig. 1) joining $m n(m o)$. Numerator diagrams can have one or more points other than $m n(0)$ each of which must be connected by a correlation line to either $m, n,(o)$, or another point.

Exchanges are shown by state lines (element 4 of fig. 1) which must form closed non-touching loops. The derivative state line in $W_{F}$ and $U_{F}$ diagrams must also be in a closed exchange loop. On momentum summation the state line $e^{i k_{i}-r_{I I}}$ becomes the familiar Slater function $l_{i j}$ :

$$
l_{i j}=\frac{3}{\left(k_{\mathrm{F}} r_{i j}\right)^{3}}\left(\sin \left(k_{\mathrm{F}} r_{i j}\right)-k_{\mathrm{F}} r_{i j} \cos \left(k_{\mathrm{F}} r_{i j}\right)\right) .
$$

An exchange loop with $(a+1)$ particles (and thus $(a+1)$ state lines) can be produced by $(a)$ pairwise exchanges. Thus there is an exchange operator:

$$
-\frac{1}{4}\left(1+O_{i j}^{\sigma}+O_{i j}^{\tau}+O_{i j}^{\sigma \tau}\right)
$$




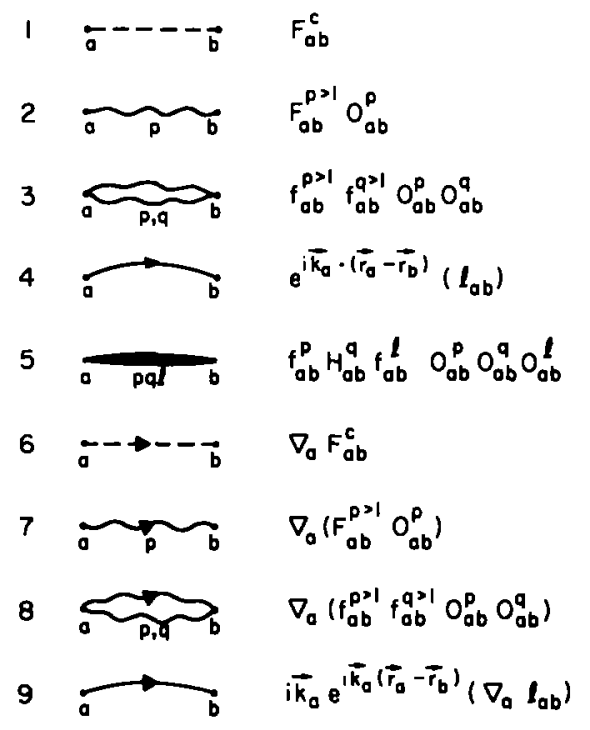

Fig. 1. Dictionary of diagrammatic elements.

associated with all but one state lines $l_{i j}$ in the loop. The line to be omitted can be chosen for convenience.

A general cluster exapnsion for noncommuting correlation operators, like $\mathscr{F}_{i}$, has been derived previously ${ }^{8}$ ). The first term in the expansion is the sum of all irreducible numerator diagrams. Additional terms coming from separable diagrams contribute only through commutators of the operators contained in $\Psi_{v}$.

The potential energy and kinetic energy terms having $\left(\nabla_{m}^{2} F_{m n}\right)$ are given by $W$-diagrams, while $U$ and $\left(W_{F}+U_{F}\right)$ respectively give the kinetic energies associated with $\left(\nabla_{m} F_{m n}\right) \cdot\left(\nabla_{m} F_{m o}\right)$, and $\left(\nabla_{m} F_{m n}\right) \cdot\left(\nabla_{m} \Phi\right)$ terms. The $\left(\nabla_{m}^{2} \Phi\right)$ terms give the Fermi gas kinetic energy $T_{\mathrm{F}}\left(=0.3 \hbar^{2} k_{\mathrm{F}}^{2} / m\right)$. We first consider the irreducible diagrams which cannot be broken into two pieces at any articulation point. Their contribution is given by the product of an integral and a $C$-factor. The integral is over all $r_{i}$ of all the functions of $r_{i j}$ represented by the lines in the diagram, while the $C$-factor takes into account the product of operators associated with the lines.

The $\Pi O_{i j}^{p}$ can always be written as:

$$
\Pi O_{i j}^{p}=C\left(\Pi O_{i j}^{p}\right)+\text { rest, }
$$

where $C\left(\Pi O_{i j}^{p}\right)$ is independent of any $\sigma_{i}$ or $\tau_{i}$, while the rest contains terms that are linear in at least one $\sigma_{i}$ or $\tau_{i}$ and goes away on spin-isospin summation. Due to the $\left(\mathscr{S} \prod_{i<i} F_{i j}\right)$ in (2.1) the operators $O_{i j}^{p}$ in a diagram can occur in various orders, and in general the $C\left(\Pi O_{i j}^{p}\right)$ depends upon the order of the operators. Let the probability of their occurring in a specific order represented by $\prod_{n} O_{i j}^{p}$ be $w_{n}$. The $C$-factor of the 
diagram is given by:

$$
\sum_{n} w_{n} C\left(\prod_{n} O_{i j}^{p}\right)
$$

The general rules for calculating $C\left(\Pi O_{i j}^{p}\right)$ are given in ref. $\left.{ }^{9}\right)$. Their calculation is simpler for the $\Pi O_{i j}^{p \leq 6}$ and hence the $E(\rho)$ of $v_{6}$ models can be calculated more easily. Henceforth, unless stated explicitly, we will assume $p \leqq 6$, and restrict ourselves to $v_{6}$ models. The $C\left(\Pi O_{i j}^{p}\right)$ in $v_{6}$ models is non-zero only when the operators $O_{i j}^{p \geq 2}$ form closed rings.

The chain equation ${ }^{10}$ ).

$$
G_{i j}=\boldsymbol{\theta}\left(X_{i k},\left(X_{k j}+G_{k j}\right)\right),
$$

offers a method to sum important diagrams in an orderly fashion. The $G_{i j}$ is the sum of all chains formed with links $X_{11} X_{12} \ldots X_{n-1, n} X_{n j}(n=1, \infty)$, while $\theta$ is an integral operator that couples the links. The links and chains in nuclear matter are denoted by $X_{x x^{\prime}, i j}^{p}$ and $G_{x x^{\prime}, i p}^{p}$ where $p$ refers to the associated operator $O_{i j}^{p}$ and $x x^{\prime}$ refer to the exchange pattern at the ends $i$ and $j$. If $i$ and $j$ are not exchanged $x x^{\prime}=\mathrm{dd} ; x x^{\prime}=\mathrm{de}$ if $i$ is not exchanged and $j$ is exchanged in a closed loop contained within the link or the chain. The $i$ and $j$ are both exchanged in closed loops inside the $G_{\text {ee, } i j}^{p}$ and $X_{e e, i j}^{p}$, while $X_{c, i j}^{p}$ and $G_{c, i j}^{p}$ have an incomplete exchange loop passing through $i$ and $j$. This classification of the $X$ and $G$ with exchange pattern was first suggested by Fantoni and Rosati ${ }^{11}$ ), though our notation is somewhat different than theirs. It is very convenient because the $X_{x x^{\prime}, i k}$ and $X_{y y^{\prime}, k j}$ cannot form a chain unless $x^{\prime} y=\mathrm{dd}$, de, ed or cc.

The $\boldsymbol{\theta}$ operators are obained from the identity:

$$
\sum_{\sigma_{k} \tau_{k}} \int \mathrm{d} \phi_{k} O_{i k}^{p} O_{k j}^{q}=\int \mathrm{d} \phi_{k} \sum_{l=1,6} \xi_{i k j}^{p q l} O_{i j}^{l} ;
$$

here $\phi_{k}$ is the azimuthal angle of $r_{i k}$ with respect to the axis $r_{i j}$. The chain equations for dd, de and ee chains in nuclear matter are of the form:

$$
\begin{aligned}
G_{x x^{\prime}, i j}^{l} & =\sum_{\substack{p, q \\
y y^{\prime}}} \Theta_{y y^{\prime}}^{p q l}\left(X_{x y, i k}^{p},\left(X_{y^{\prime} x^{\prime}, k j}^{q}+G_{y^{\prime} x^{\prime}, k j}^{q}\right)\right), \\
\Theta_{y y^{\prime}}^{p q l} & =\int d^{3} r_{k} \xi_{i j k}^{p q l} \quad \text { if } \\
& =0 \text { otherwise. }
\end{aligned}
$$

The $\xi_{i j k}^{p q l}$ are given in ref. ${ }^{8}$ ).

The diagrams summed by (2.10) depend upon the chosen link functions $X_{x x^{\prime}}^{p}$. In the FHNC/SOC approximation the links are allowed to contain hypernets of $G_{x x^{\prime}}^{\mathrm{c}}$, 
but only single chains of $O^{p \geq 2}$ operators. Thus the $X_{d d}^{p}$ for example become:

$$
\begin{aligned}
X_{\mathrm{dd}}^{c} & =\left\{f^{c^{2}}+\sum_{p=2,6} A^{p}\left(f^{p}+f^{c} G_{d d}^{p}\right)^{2}\right\} \exp \left(G_{\mathrm{dd}}^{c}\right)-1-G_{\mathrm{dd}}^{\mathrm{c}}, \\
X_{\mathrm{dd}}^{p \geq 2} & =\left(F^{p}+f^{c^{2}} G_{\mathrm{dd}}^{p}\right) \exp \left(G_{\mathrm{dd}}^{\mathrm{c}}\right)-G_{\mathrm{dd}}^{p},
\end{aligned}
$$

where the $A^{p}$ are defined as:

$$
C\left(O_{i j}^{p} O_{i j}^{q}\right)=A^{p} \delta_{p q}
$$

The FHNC/SOC approximation should be reasonable when $F^{p \geq 2}, f^{p \geq 2}$ and $G_{x x^{\prime}}^{p \geq 2}$ are $\ll 1$.

The $G_{x x^{\prime}}^{\mathrm{c}}$ sum all the FHNC diagrams due to the central correlation $f^{c}$ plus diagrams that have closed single operator rings (SOR) as illustrated in fig. 2.1,2. The $G_{x x^{\prime}}^{\mathrm{c}}$ also contain diagrams with touching SOR's ( 2.3 for example) but their $C$-part is

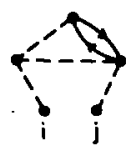

2.1

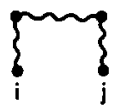

2.4

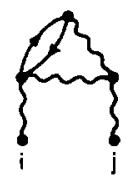

2.7

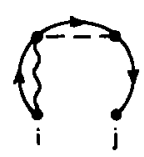

2.10

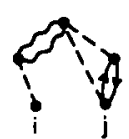

2.2

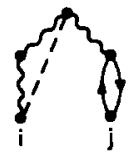

2.5

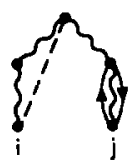

2.8

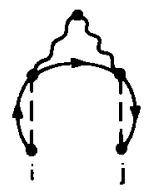

2.11

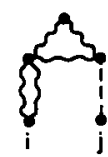

2.3

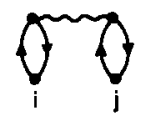

2.6

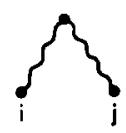

2.9

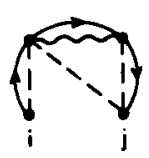

2.12

Fig. 2. Examples of chain diagrams. 
grossly approximated. $\left.{ }^{8}\right)$ However, when $f^{p \geq 2}$ and $G_{x x^{\prime}}^{p \geq 2}$ are $\ll 1$ these ill-treated diagrams should be very small.

Some of the simpler diagrams summed by the FHNC/SOC $G_{x x^{\prime}}^{p=2}, x x^{\prime}=$ dd, de and ee are shown in fig. 2.4-6; these can contain any number of $G_{x x^{\prime}}^{\mathrm{c}}$ dressings which are not explicitly shown. Due to the SOC restriction diagrams of type 2.7-8 are not included in the $G_{x x^{\prime}}^{p \geq 2}$; the operator algebra of these diagrams is non-trivial. However, when $F^{p \geq 2}$ and $G_{x x^{\prime}}^{p \geq 2}$ are both $\ll 1$ the neglected diagrams are much smaller than those summed. It should be noted smallness of the magnitude of $F^{p \geq 2}$ does not imply that the $G_{d d}^{p}$ diagram 2.4 is smaller than 2.9 , when $f^{p}$ have a range $>r_{0}$. In general when $\left(d / r_{0}\right)^{3}>1$ the chain diagrams need to be summed. All but one of the exchange lines in a $G_{\mathrm{cc}}$ chain carry an exchange operator, thus in the SOC approximation the $G_{c c}^{p \geq 2}$ can have at most one $F^{p}$ or $G_{d d}^{p}$ link, as illustrated in diagrams 2.10-12, which merely "fills up the gap". The detailed equations for $G_{\mathrm{cc}}^{p}$ are given in ref. ${ }^{8}$ ).

\section{Calculation of the energy}

It is convenient to define three matrices $K^{i j k}, L^{i j k}$ and $D_{i j}$ to calculate the energy from the chain functions $G_{x x^{\prime}}^{p}$. The $K^{i j k}$ is defined as:

$$
O_{m n}^{i} O_{m n}^{i}=\sum_{k} O_{m n}^{k} K^{i j k} \text {. }
$$

From eqs. (3.1), (2.9) and (2.14) we obtain

$$
\int \mathrm{d} \phi_{1} C\left(O_{m n}^{i} O_{m n}^{\prime} O_{m 1}^{l^{\prime}} O_{n 1}^{l^{*}}\right)=\int \mathrm{d} \phi_{1} \sum_{l} K^{i l i l} \xi_{m 1 n}^{\prime \prime l \mid l} A^{l} .
$$

The $L^{l i k}$ is defined as:

$$
\begin{gathered}
\int \mathrm{d} \phi_{1} C\left(O_{m n}^{i} O_{m 1}^{l^{\prime}} O_{m n}^{j} O_{n 1}^{l^{\prime \prime}}\right)=\int \mathrm{d} \phi_{1} \sum_{l} L^{i l l} \xi_{m 1 n}^{l^{\prime} l^{\prime \prime} l}, \\
L^{i j l}= \pm K^{l j l} A^{l} .
\end{gathered}
$$

The + sign applies when

$$
C\left(O_{m n}^{i}\left[O_{m n}^{i}, O_{m 1}^{l^{\prime}}\right] O_{n 1}^{l^{\prime \prime}}\right)=0,
$$

and the - sign when

$$
C\left(O_{m n}^{i}\left\{O_{m n}^{i}, O_{m 1}^{l^{\prime}}\right\} O_{n 1}^{l^{\prime \prime}}\right)=0 \text {. }
$$

In the $v_{6}$ problem it can be shown ${ }^{7}$ ) that either (3.5) or (3.6) is valid if $K^{i l l}$ and $\xi_{m l n}^{\prime \prime l l}$ are nonzero. The $D_{l j}$ is used to calculate the contribution of separable diagrams:

$$
C\left(O_{m n}^{i}\left[O_{m 1}^{j^{\prime}}, O_{m n}^{i^{\prime}}\right] O_{m 1}^{i}\right)=\delta_{i t}, \delta_{i j^{\prime}} A^{i} A^{i} D_{i j}
$$

Useful symmetry properties of the $K$ - and $L$-matrices are reviewed in ref. ${ }^{7}$ ). 
The energy given by the sum of $W, U, W_{\mathrm{F}}$ and $U_{\mathrm{F}}$ diagrams can be calculated within the FHNC/SOC approximation with the help of matrices $A, K, L$ and the $G_{x x^{\prime}}^{p}$. We illustrate the method by calculating some of the contributions to $W$; the complete calculation is given in ref. ${ }^{7}$ ).

The $W$-diagrams are divided into four classes $W_{0}, W_{c}, W_{1}$ and $W_{c}$ as illustrated in fig. 3.1-4. Diagrams of any class can have any number of $G_{x x^{\prime}, m n}^{c}$ chains, and any exchange pattern, neither of which are shown in fig. 3 . The only operators in $W_{0}$

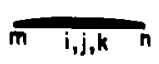

$w_{0}(3.1)$

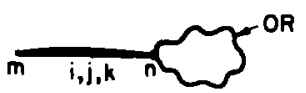

$W_{s}(3.3)$

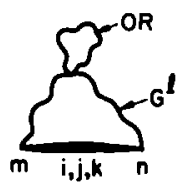

(3.5)

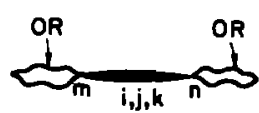

(3.7)

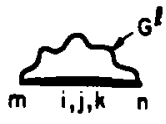

$w_{c}(3.2)$

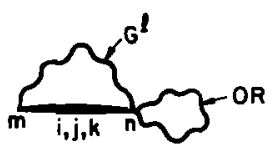

$W_{C S}(3.4)$

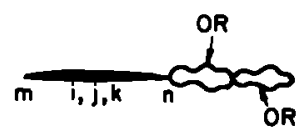

(3.6)

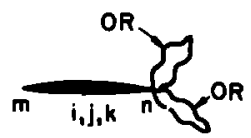

(3.8)

Fig. 3. Classification of $\boldsymbol{W}$ diagrams.

diagrams are the $O_{m n}^{l}, O_{m n,}^{i}, O_{m n}^{k}$ associated with the $\mathscr{F}_{m n,}^{\dagger}, H_{m n}$ and $\mathscr{F}_{m n}$, and possibly the $O_{m n}^{n}$ associated with the exchange of $m n$. Please avoid confusion between operator labels $i, j, k, l, m, n \ldots$ and particle labels $m, n, o \ldots$ We get:

$$
\begin{aligned}
W_{0}=\frac{1}{2} \rho & \sum_{i, k=1,6} \int d^{3} r f^{l} H^{j} f^{k} h^{c} K^{l j k} A^{k}\left(\left(1+G_{d o}^{c}\right)^{2}+G_{\odot e}^{c}\right) \\
& -\frac{1}{8} \rho \sum_{h, j=1,6} \sum_{\substack{n=1,4 \\
m=1,6}} \int d^{3} r f^{i} H^{j} f^{k} h^{c} \mathscr{L}^{2} K^{n i m} K^{j k m} A^{m},
\end{aligned}
$$


where $h^{c}$ takes into account the hypernetted $G_{d d}^{c}$ chains:

$$
h^{\mathrm{c}}=\exp \left(G_{\mathrm{dd}}^{\mathrm{c}}\right) \text {, }
$$

the generalized Slater function $\mathscr{L}$ contains the $G_{\mathrm{c}}^{\mathrm{c}}$ chains:

$$
\mathscr{L}(r)=-l\left(k_{\mathrm{F}} r\right)+4 G_{c \mathrm{c}}^{\mathrm{c}}(r),
$$

and the $\boldsymbol{K}$ and $\boldsymbol{A}$ matrices give the $\boldsymbol{C}$-factor.

The $W_{c}$ diagrams have the operators $O_{m 1}^{l^{\prime}} O_{12}^{p} O_{23}^{q} \ldots O_{a n}^{l^{*}}$ in $G_{m n}^{l=2}$, where $1,2,3 \ldots a$ are the internal points in the chain, in addition to the operators $O_{m n}^{\prime}, O_{m n}^{\prime}, O_{m n}^{k}$ and possibly $O_{m n}^{n}$. Their $C$-part depends upon the order of the operators $l^{\prime}, l^{\mu}, i, j, k$ and $n$ (we denote $O_{m n}^{l}$ by $i$ for brevity), while the positions of operators $p, q \ldots$ in the middle of the chain are irrelevant. The $C$-factor of $W_{c}$ diagrams has to be calculated with eq. (2.7). The probabilities $w$ depend upon the exchange pattern $x x^{\prime}$ and so the $W_{\mathrm{c}}\left(x x^{\prime}\right)$, which give the contribution of $G_{x x^{\prime}, m n}^{l=2}$ to $W_{\mathrm{c}}$, have to be separately evaluated.

Let us consider $W_{\mathrm{c}}(\mathrm{de})$ diagrams in which the operator $l^{n}$ comes from exchange and may be placed at either end of the product. The $l^{\prime}$ may be associated with $F_{m 1}$ or $\mathscr{F}_{m 1}^{\dagger}$ and so we have to consider orders: $l^{\prime \prime} i j k l^{\prime}, l^{\prime \prime} i j l^{\prime} k, l^{\prime \prime} i l^{\prime} j k$ and $l^{\prime \prime} l^{\prime} i j k$ all occurring with probability $w=\frac{1}{4}$. The $C$-part in these orders can be calculated in terms of the $A-, K-, L-$ and $\xi$-matrices, and we obtain:

$$
\begin{aligned}
W_{\mathrm{c}}(\mathrm{de})=\rho \sum_{\text {Ll,k=1,6 }} \sum_{\substack{i=2,6 \\
m-1,6}} \int \mathrm{d}^{3} r f^{i} H^{i} f^{k} G_{\mathrm{de}}^{l} \frac{1}{4}\left(2 A^{m} K^{i j m} K^{k l m}\right. \\
\\
\left.+K^{i j m} L^{k l m}+K^{i k m} L^{i l m}\right) h^{\mathrm{c}}\left(1+G_{\mathrm{de}}^{\mathrm{c}}\right),
\end{aligned}
$$

all the $\xi$-functions being contained in the $G_{\text {de }}^{l}$.

In the $W_{8}$ diagrams (fig. 3.3) the operator ring (OR) that can be separated at the articulation point $n$ can be formed either with an $f_{n 1}^{l} f_{n 1}^{l}$ element $(l \geqq 2)$ or with a $G_{x x^{\prime}, n 1}^{l} F_{n 1}^{l}$. The contribution of these separable diagrams is proportional to the difference between the $C$-factor of the connected diagram and the product of the $C$-factors of the two separated pieces. Let us consider the simplest of the $W_{\text {. }}$ diagrams in which there are no central $G_{x x^{\prime}}^{e}$ dressings or exchanges, and the OR is $f_{n 1}^{l} f_{n 1}^{l}$. The difference between $C$-factors is then:

$$
{ }_{4}^{1} C(\{i, l\} j\{i, l\})-C(i j k) C(l l)=\frac{1}{4} K^{l i k} A^{k} A^{l}\left(D_{u}+D_{j l}+D_{k l}\right) .
$$

In this way we can calculate the operator parts of $W_{3}$ diagrams in terms of $A ; K$ - and $D$-matrices.

It is possible to treat $W_{2}$ diagrams (3.3) exactly as vertex corrections at vertices $m$ and $n$ of $W_{0}$ diagrams (3.1) ${ }^{7}$ )] The diagrams of type (3.5) and (3.6) can be treated as corrections at the vertices within $G_{n 1}^{l}$. Vertex factors can be easily inserted in the chain eq. $\left.2.10-11^{8}\right)$ ] The $W_{a}$ diagrams (3.4), and those of type (3.7) can also be summed with a very reasonable accuracy of $\simeq 0.2 \mathrm{MeV}$ as corrections to diagrams 
(3.2) and (3.3) respectively. However, the diagrams of type (3.8) are neglected at this stage, the magnitude of their contribution could be $\simeq 5-10 \%$ of $W_{4}$.

\section{Results}

The Reid $v_{6,8}$ and BJ-II $v_{6,8}$ models are obtained by expressing the ${ }^{1} \mathrm{~S}_{0,}{ }^{3} \mathrm{~S}_{1}-$ ${ }^{3} \mathrm{D}_{1},{ }^{1} \mathbf{P}_{1}$, and ${ }^{3} \mathbf{P}_{2}-{ }^{3} \mathbf{F}_{2}$ poteritials in the Reid ${ }^{12}$ ) and Bethe -Johnson-II ${ }^{13}$ ) interaction models as $\sum_{l} v^{l}(r) O^{i-1,8}$. The $(L \cdot S)$ potentials are neglected in the $v_{6}$ models. The $\mathrm{HJ} v_{6}$ model is obtained by neglecting the $(L \cdot S)$ and quadratic spin-orbit terms in the Hamada-Johnson ${ }^{14}$ ) potential, while the Gammel-Thaler $5200^{15}$ ) (GT5200) potential is itself of a $v_{6}$ form.

The $\sigma \tau$ and $t \tau$ correlations and chains are most important in nuclear matter, and the $f^{\sigma r}, f^{\pi t}, G_{d e}^{\sigma r}$ and $G_{d e}^{t r}$ in the Reid $v_{6}$ model are shown in fig. 4 for $d=2.25 r_{0}$, and $\beta_{l-2,6}=1$. The $G^{l \geq 2}$ are very long ranged, and particularly the $G_{x x^{\prime}}^{\sigma r}$ are comparable to $f^{i \neq 2}$. For such cases the $\boldsymbol{\theta}\left(X_{i k}, G_{k j}\right)$ term in $G_{i j}$ [eq. (2.10)] is important, and it is necessary to solve the integral chain equations. However, the $f^{l \geq 2}$ and $G^{l \geq 2}$ are very small in magnitude and thus the SOC approximation should be valid.

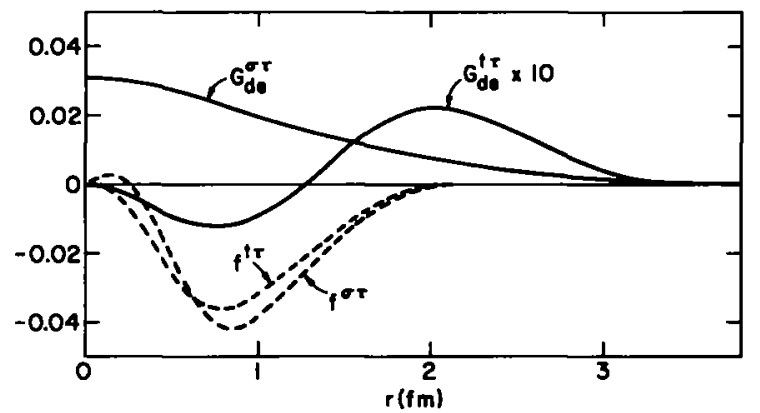

Fig. 4. The $f^{\sigma r}, f^{t r}, G_{d 0}^{\sigma r}$ and $G_{d o}^{\sigma}$ in nuclear matter at $k_{F}=1.6 \mathrm{fm}^{-1}, d=2.25 r_{0}, \beta_{l=1}=1$.

The energy is more sensitive to $\beta_{t r}$ and $d$ than to $\beta_{o m}$ and it is very insensitive to $\beta_{\sigma}, \beta_{r}$ and $\beta_{t}$. The equilibrium value of $\beta_{t r}$ is close to unity, however that of $\beta_{\sigma r}$ is generally $<1$. The $E\left(k_{\mathrm{F}}, d, \beta_{i}\right)$ for Reid (BJ-II) $v_{6}$ model at its minimum is given in table $1(2)$. The $f^{\sigma}, f^{\tau}$ and $f^{\sigma r}$ lower the energy by $2(5) \mathrm{MeV}$. There is a very large cancellation between the two-body and many-body cluster contributions (MBCC) due to $f^{\sigma}, f^{\tau}$ and $f^{\sigma \tau}$. The largest contribution of $f^{t}$ and $f^{t \tau}$ to MBCC is via the $W_{s}$. The $E(\rho)$ of these models is shown in fig. 5, along with the "experimental" $E(\rho)$ and crude estimates of that of the $v_{8}$ models $\left.{ }^{7}\right)$. The difference between $v_{6}$ and $v_{8}$ is significant and indicates the importance of $(\boldsymbol{L} \cdot S)$ contributions to nuclear energy. Unfortunately, the $v_{8}$ models are much harder to treat accurately.

The recent ${ }^{18}$ ) Reid $v_{6}$ model results obtained with the Brueckner-Bethe-Goldstone (BBG) method are also shown in fig. 5. The curve labled BBG includes two- 
TABLE 1

Reid $v_{6} E\left(k_{\mathrm{F}}=1.6 \mathrm{fm}^{-1}, d=2.5 r_{0}\right)$ in $\mathrm{MeV}$

\begin{tabular}{|c|c|c|c|c|}
\hline $\boldsymbol{\beta}_{\mathbf{I}}$ & $\beta_{i}=1$ & $\begin{array}{c}\beta_{t, \pi t}=1 \\
\beta_{i=2,4}=0.4\end{array}$ & $\begin{array}{c}\beta_{L, T}=1 \\
\beta_{i=2,4}=0\end{array}$ & $\beta_{i z 2}=0$ \\
\hline$T_{F}$ & 31.85 & 31.85 & 31.85 & 31.85 \\
\hline 2-body & -65.76 & -59.16 & -52.75 & -10.75 \\
\hline$W_{0}(\mathrm{MB})$ & -6.91 & -6.30 & -5.28 & 1.97 \\
\hline$W_{c}$ & 13.02 & 6.46 & 0.77 & $\mathbf{0}$ \\
\hline$W_{1}$ & 18.30 & 12.21 & 10.35 & $\mathbf{0}$ \\
\hline$w_{\mathbf{c}}$ & -2.97 & -1.01 & 0.05 & 0 \\
\hline$W_{\mathrm{F}}(\mathbf{M B})$ & -1.07 & -0.20 & 0.48 & 0.18 \\
\hline \multicolumn{5}{|c|}{$+U_{F}$} \\
\hline$U$ & -0.91 & -0.80 & -0.60 & 1.93 \\
\hline$E$ & -14.45 & -16.95 & -15.13 & 25.18 \\
\hline
\end{tabular}

TABLE 2

\begin{tabular}{|c|c|c|c|c|}
\hline \multicolumn{5}{|c|}{ BJ-II $v_{6} E\left(k_{F}=1.2 \mathrm{fm}^{-1}, d=2.25 r_{0}\right)$ in MeV } \\
\hline $\boldsymbol{\beta}_{\boldsymbol{i}}$ & $\beta_{i}=1$ & $\begin{array}{c}\beta_{\text {Lit }}=1.1 \\
\beta_{i-2,4}=0.6\end{array}$ & $\begin{array}{l}\beta_{i, \pi}=1.1 \\
\beta_{i-2,4}=0\end{array}$ & $\boldsymbol{\beta}_{t \geq 2}=0$ \\
\hline$T_{\mathbf{F}}$ & 17.92 & 17.92 & 17.92 & 17.92 \\
\hline 2-body & -39.70 & -35.48 & -24.17 & -8.40 \\
\hline$W_{0}(\mathrm{MB})$ & -0.82 & -0.98 & -0.80 & -0.12 \\
\hline$W_{c}$ & 3.43 & 1.85 & -0.58 & 0 \\
\hline$w_{\mathbf{a}}$ & 14.68 & 10.73 & 6.79 & 0 \\
\hline$W_{\mathbf{a}}$ & -1.03 & -0.56 & 0.25 & 0 \\
\hline$W_{\mathbf{F}}(\mathrm{MB})$ & 0.62 & 0.42 & 0.12 & -0.01 \\
\hline \multicolumn{5}{|c|}{$+U_{F}$} \\
\hline $\boldsymbol{U}$ & -0.19 & -0.54 & -0.78 & 0.45 \\
\hline $\boldsymbol{E}$ & -5.09 & -6.64 & -1.25 & 9.83 \\
\hline
\end{tabular}

and three-hole-line contributions, and estimates of four-hole-line contributions. It is significantly below the LOBT curve which includes only the two-hole-line contribution. A plausible estimate of errors in the $v_{6}$ results at $k_{\mathrm{F}}=1.6 \mathrm{fm}^{-1}$ may be made as follows. The error due to truncating the chain summations in the present variational calculation is plausibly given by the magnitude of $W_{\mathrm{as}}$ and is $\sim 1 \mathrm{MeV}$. However, we expect the $E\left(k_{\mathrm{F}}=1.6 \mathrm{fm}^{-1}\right)$ to decrease by $\sim 1 \mathrm{MeV}$ if $k$-dependent terms are included in the correlation operator $F$, which gives $\Delta E_{\text {var }} \simeq+0$ to $-2 \mathrm{MeV}$. The error in BBG $E\left(k_{F}=1.6 \mathrm{fm}^{-1}\right)$ is $\left.\left.\pm 2 \mathrm{MeV}^{18}\right)\right]$. The BBG and variational results are certainly in agreement within these errors at $k_{F}=1.6$ and $1.8 \mathrm{fm}^{-1}$. At $k_{\mathrm{F}}=$ $1.4 \mathrm{fm}^{-1}$ the difference between the two calculations is marginally larger than the estimated errors.

The $E\left(k_{\mathrm{F}}=1.3 \mathrm{fm}^{-1}\right)$ in Reid, BJ-II, HJ and GT-5200 $v_{6}$ models are compared in table 3, and the $E(\rho)$ of $\mathrm{HJ}$ and GT-5200 models are compared with the results of 


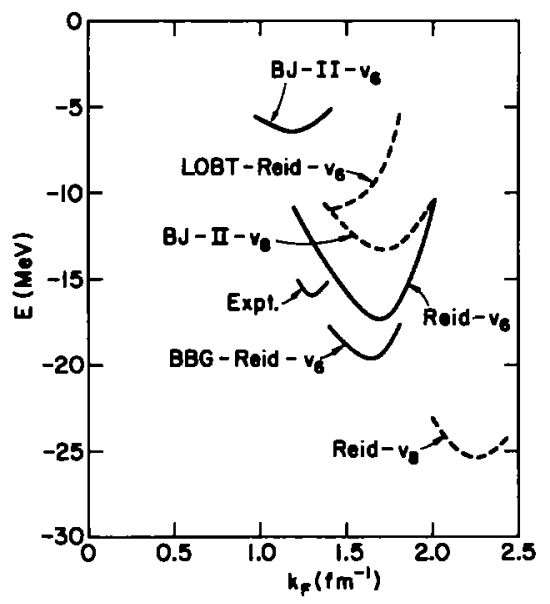

Fig. 5. A comparison of the calculated energies for Reid and BJ models with the "experiment" and the results of calculations based on the Brueckner-Bethe-Goldstone method.

TABLE 3

$E_{\mathrm{min}}\left(k_{\mathrm{F}}=1.3 \mathrm{fm}^{-1}\right)$ for various potentials in $\mathrm{MeV}$

\begin{tabular}{|c|c|c|c|c|}
\hline Model & Reid $v_{6}$ & $\mathrm{BJ}-\mathrm{II} v_{6}$ & HJ $v_{6}$ & GT-5200 \\
\hline$d / r_{0}$ & 2.25 & 2.25 & 2.35 & 2.42 \\
\hline$\beta_{i=2,4}$ & 0.4 & 0.6 & 0.5 & 0.75 \\
\hline$\beta_{i-5,6}$ & 1.0 & 1,1 & 1.0 & 1.0 \\
\hline$T_{F}$ & 21.03 & 21.03 & 21.03 & 21.03 \\
\hline 2-body & -42.28 & -37.55 & -37.04 & -50.28 \\
\hline$W_{0}(\mathrm{MB})$ & -0.21 & -3.96 & -0.15 & 1.77 \\
\hline$W_{c}$ & 2.79 & 4.01 & 2.31 & 3.43 \\
\hline$W_{\text {s }}$ & 6.44 & 11.71 & 5.19 & 6.83 \\
\hline$W_{a}$ & -0.42 & -0.87 & -0.35 & -0.66 \\
\hline$W_{F}(\mathbf{M B})$ & 0.26 & -0.17 & 0.23 & 0.69 \\
\hline \multicolumn{5}{|l|}{$+U_{F}$} \\
\hline $\boldsymbol{U}$ & -0.59 & -0.76 & -0.23 & -0.32 \\
\hline$E$ & -12.98 & -6.56 & -9.01 & -17.51 \\
\hline
\end{tabular}

Benhar et al. (BCFR) ${ }^{16}$ ) and Kurten et al. ${ }^{17}$ ) (KRC) in fig. 6. The KRC energies are much lower than ours, however they truncate the cluster expansion at the three-body level, and the second-order correlated basis perturbation term at the two-body level. Probably a more accurate calculation with their approach will give higher energies. Our results are in fair agreement with those of BCFR, whose calculation is probably closest to ours in principle. However, there are large differences between the present and the BCFR calculation, particularly in the treatment of $W_{c}$ and $W_{s}$. Thus the agreement is very encouraging but not yet fully understood. 


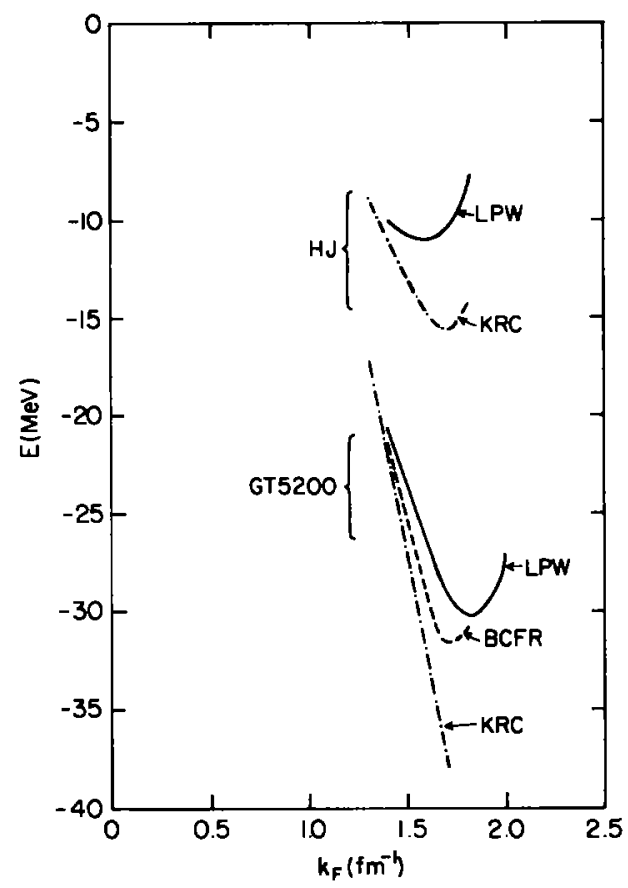

Fig. 6. A comparison of the results of variational calculations for the HJ $v_{6}$ and GT5200 modeln.

Using a somewhat different wave function Owen ${ }^{2}$ ) has calculated the $E(\rho)$ of model $v_{3}$ of neutron matter which contains the $v^{\sigma}$ and $f^{\sigma}$. With his choice of the variational wave function he could sum all hypernetted operator chain diagrams, and thus obtain presumably reliable upperbounds for the $E(\rho)$. Our FHNC/SOC results (table 4) are very similar to his and thus indicate that SOC is a good approximation. Owen also does a calculation he calls "SOC" with which he fails to obtain a variational minimum in $E(d)$. However, we believe that his "SOC" calculation is significantly different from ours.

TABLE 4

$v_{3} E\left(\rho, d, \beta_{\sigma}\right)$ in units of fm and $\mathrm{MeV}$

\begin{tabular}{cccccc}
\hline$\rho$ & $E_{\min }$ & $\left(d / r_{0}\right)_{\min }$ & $\left(\beta_{\sigma}\right)_{\min }$ & $E\left(d_{\min ,} \beta_{\sigma}=0\right)$ & $E($ Owen $)$ \\
\hline 0.17 & 17.4 & 1.9 & 0.8 & 17.8 & 17.0 \\
0.3 & 28.8 & 2.3 & 0.5 & 29.2 & 28.2 \\
0.4 & 39.9 & 2.4 & 0.45 & 40.3 & 38.8 \\
\hline
\end{tabular}

The $v_{3}$ is a very difficult model to test the effect of non-central correlations. The $f^{\sigma}$ has a very small effect on its $E(\rho)$ (table 4$)$ due to very large cancellations: at $\rho=0.3 \mathrm{fm}^{-3}$ the $E\left(\beta_{\sigma}=0, .5\right)$ are 29.2 and $28.8 \mathrm{MeV}$, while the $W_{\mathrm{c}}\left(\beta_{\sigma}=0, .5\right)$ are 0 
and 7.4 $\mathrm{MeV}$ respectively. The error in the $E(\rho)$ of our calculation, as estimated from the magnitude of $W_{\mathrm{cs}}$, is $\simeq 0.4 \mathrm{MeV}$ at $\rho=0.3 \mathrm{fm}^{-3}$; it is much smaller than leading terms in the energy such as $W_{c}$, but it is still of the same order as the net gain in energy due to $f^{\sigma}$.

The authors wish to thank Dr. B. D. Day and Dr. J. C. Owen for communicating their results to us.

Note added in proof: Recent calculations of the Reid and BJ $v_{8}$ models by Lagaris and Pandharipande indicate that the difference between the $v_{6}$ and $v_{8}$ model energies is much smaller than shown in fig. 5 .

\section{References}

1) V. R. Pandharipande, R. B. Wiranga and B. D. Day, Phys. Lett. 59B (1975) 205

2) J. C. Owen, preprint (1978)

3) K. E. Schmidt and V. R. Pandharipande, Proc. of the Int. Conf on Recent progreas in many-body theories, Trieste, Nucl. Phys. A328 (1979) 240

4) L. J. Lantto and P. J. Siemens, Phys. Lett. 68 (1977) 308; Nucl. Phys. A317 (1979) 55

5) V. R. Pandharipande and H. A. Bethe, Phys. Rev. C7 (1973) 1312

6) V. R. Pandharipande and K. E. Schmidt, Phys. Rev. A1s (1977) 2488

7) R. B. Wiringa and V. R. Pandharipande, Nucl. Phys. A317 (1979) 1

8) R. B. Wiringa and V. R. Pandharipande, Nucl. Phys. A299 (1978) 1

9) V. R. Pandharipande and R. B. Wiringa, Nucl. Phys. A266 (1976) 269

10) J. M. J. Van Leeuwen, J. Groeneveld and J. deBoer, Physica 25 (1959) 792

11) S. Fantoni and S. Rosati, Nuovo Cim. 25A (1975) 593

12) R. V. Reid, Ann. of Phys. so (1968) 411

13) H. A. Bethe and M. B. Johnson, Nucl. Phys. $\mathbf{A 2 3 0}$ (1974) 1

14) T. Hamada and I. D. Johnston, Nucl. Phys. 34 (1962) 382

15) J. L. Gammel and R. M. Thaler, in Progress in elementary particle and cosmic ray physics ed J. G. Wilson and S. A. Wouthuysen (North-Holland, Amaterdam, 1960), vol. V

16) O. Benhar, C. Ciofi degli Atti, S. Fantoni and S. Rowati, Phys. Lett 70B (1977) 1

17) K. E. Kurten, M. L. Ristig and J. W. Clark, Phys. Lett 74B (1978) 153; Nucl. Phys. A317 (1979) 87

18) B. D. Day private communication (Sept. 1978) 